\title{
Editorial
}

\section{LOS PUNTOS MOTORES: UNA EXPRESIÓN MORFOLÓGICA CON AMPLIAS APLICACIONES CLÍNICAS QUE NO SE HA ESTUDIADO SUFICIENTEMENTE}

\author{
Luis E. Ballesteros \\ Departamento de Ciencias Básicas, área de morfofisiologia,,Universidad Industrial de \\ Santander, Bucaramanga, Colombia
}

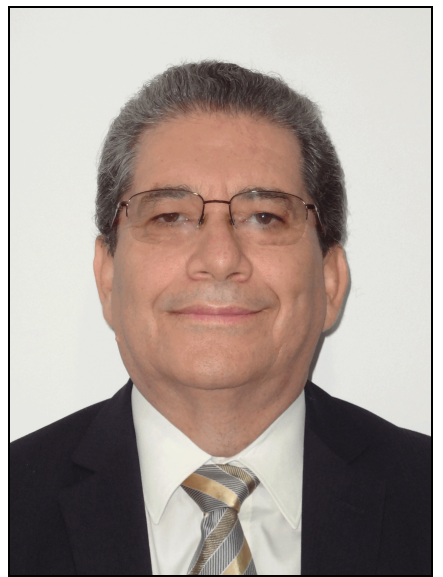

Morfológicamente, el punto motor (PM) corresponde al sitio en el que el ramo motor de un nervio ingresa al vientre muscular $\mathrm{Al}$ ingresar al músculo, el ramo motor se distribuye de forma amplia, realizando recorridos intrafasciculares que establecen conexiones con las fibras musculares que dan lugar a las placas neuromusculares, unidades funcionales que permiten la transmisión del impulso sináptico desde el nervio hacia el tejido contráctil objetivo.

En clínica, el PM corresponde a la zona de la piel en la cual se puede lograr contracción muscular con una mínima estimulación eléctrica; por lo que pueden identificarse mediante procedimientos no invasivos, por medio de electrodos ubicados en la superficie de la piel que generan pulsos eléctricos que determinan la contracción muscular. La ubicación de los ramos y PM representan entonces un referente anatomofuncional importante para el uso de herramientas diagnósticas o de intervención de alto impacto en evaluación, diagnóstico, rehabilitación, fisiatría, ortopedia y cirugía plástica, los cuales basan su quehacer en adecuadas ubicaciones topográficas que conducen a una precisión exitosa de los procedimientos realizados.

Los estudios relacionados con la distribución motora en las diferentes regiones topográficas son en términos generales escasos, y con mayor énfasis en algunas regiones como la braquial anterior y posterior de la pierna, mientras que en otras como el dorso la información es de un carácter general y limitadas a algunos grupos poblacionales. Las expresiones variantes de las ramas motoras, así como sus características morfo métricas relacionadas con los sitios óseos de referencia que permitan su fácil localización, no han sido incorporadas en los textos de anatomía por lo que los estudiantes de pre y posgrados no acceden fácilmente a este tipo de información.

La distribución de las ramas motoras es compleja, algunas se expresan como troncos únicos de longitud y trayectoria variable que se ponen en contacto con los vientres musculares correspondientes directamente o dividiéndose previamente en dos o tres ramas que se distancias entre si antes de constituirse en PM; Igualmente, en algunas regiones se encuentran grandes ramas motoras primarias que a su vez van originando ramas que se proyectan a 
músculos del mismo compartimiento topográfico y que finalizan como rama única o múltiple.

Sunderland et al (1985) han cuantificado en caucásicos las ramas y PM para cada uno de los músculos de las extremidades superiores e inferiores, información que permite realizar un mapeo motor de estos segmentos corporales; sin embargo, dado el pequeño universo de la muestra estudiada que excluye a las expresiones variantes y la carencia de datos morfo métricos que permitan localizar puntualmente a los PM, limitan su utilización en las diversas aplicaciones clínicas y quirúrgicas. A pesar de la existencia de detallados estudios, aunque limitados a compartimentos corporales específicos (Seidel et al 1996; Apaydin et al, 2008; Bowden y McNulty, 2012; Olave et al, 2012), se requiere que en los diversos grupos poblacionales se realicen trabajos que determinen la configuración de los PM en razón a las diferencias muchas veces marcadas de la talla corporal y a la variabilidad anatómica determinadas por los polimorfismos genéticos de las diferentes etnias, que impiden extrapolar datos obtenidos de otros grupos poblacionales, lo que conduce a la necesidad de tener información propia de referencia.

La detallada descripción de los PM permite establecer el área de dispersión de la inervación, y su distancia con relación a sitios de referencia, para cada uno de los vientres musculares estudiados, información con variadas aplicaciones clínicas y quirúrgicas como son los procedimientos de electro diagnóstico, electroestimulación, bloqueos nerviosos en procedimientos anestésicos, infiltración de toxina botulínica en pacientes con espasticidad severa y en los abordajes quirúrgicos en las áreas de cirugía plástica (Lukban et al, 2009; Thomas y Mackinnon, 2010). En las electromiografías, los electrodos de estimulación deben colocarse por fuera del área de dispersión de los PM, mientras que las técnicas de electroterapia, especialmente la estimulación eléctrica neuromuscular requiere la aplicación de los electrodos sobre el área donde están los respectivos PM. Quienes hacen estos procedimientos deben realizar en cada paciente ejercicios previos de ensayo con la aplicación de los electrodos hasta identificar las áreas de mayor estimulación eléctrica y que corresponden a los PM. Esta actividad empírica podría subsanarse y mejorar su eficacia terapéutica con una adecuada información que los estudios anatómicos puedan suministrar sobre la ubicación de los PM.

El conocimiento de los PM es fundamental para el planeamiento de cirugías reconstructivas, específicamente de trasferencias nerviosas y de colocación de injertos, en donde la adecuada preparación y longitud de las ramas donantes o de las receptoras son determinantes para el éxito de estos procedimientos (Thomas y Mackinnon, 2010).

Los PM son un tema no agotado en la investigación anatómica por lo que se requiere extender su estudio a todas las regiones corporales, en los diversos grupos poblacionales, que genere información valiosa orientada a optimizar el ejercicio de múltiples aplicaciones clínicas.

\section{Referencias:}

Apaydin N, Loukas M, Kendir S, Tubbs R, Jordan R, Tekdemir I, Elhan A. 2008. The precise localization of distal motor branches of the nerve in the deep posterior compartment of the leg. Surg Radiol Anat; 30: 291-95.

Bowden J, McNulty P. 2012. Mapping the motor point in the human tibialis anterior muscle. Clinical Neurophysiology; 123: 386-92.

Lukban MB, Rosales RL, Dressler D. 2009. Effectiveness of botulinum toxin $A$ for upper and lower limb spasticity in children with cerebral palsy: a summary of evidence. J Neural Transm; 116: 319-31.

Olave E, Retamal P, Galaz C, Cruzat C. 2012. Inervación y Localización Biométrica de los Puntos Motores del Músculo Tríceps Sural. Int J Morphol; 30: 1056-60

Seidel P, Seidel G, Gans B, Dijkers M. 1996. Precise Localization of the Motor Nerve Branches to the Hamstring Muscles: An Aid to the Conduct of Neurolytic Procedures. Arch Phys Med Rehabil; 77: 1157-60.

Sunderland, S. 1985. Nervios periféricos y sus lesiones. Barcelona: Salvat Editores, S.A.

Thomas T, Mackinnon S. 2010. Nerve transfers: indications, techniques and outcomes. J Hand Surg Am; 35: 332-41. 